\title{
Identification of complex nonlinearities using cubic splines with automatic discretization
}

\author{
T. Dossogne, J.P. Noël and G. Kerschen \\ Space Structures and Systems Lab \\ Aerospace and Mechanical Engineering Department \\ University of Liège \\ 9, Allée de la Découverte (B52/3), 4000 Liège, Belgium \\ Email: tdossogne, jp.noel, g.kerschen@ulg.ac.be
}

\begin{abstract}
One of the major challenges in nonlinear system identification is the selection of appropriate mathematical functions to model the observed nonlinearities. In this context, piecewise polynomials, or splines, offer a simple and flexible representation basis requiring limited prior knowledge. The generally-adopted discretization for splines consists in an even distribution of their control points, termed knots. While this may prove successful for simple nonlinearities, a more advanced strategy is needed for nonlinear restoring forces with strong local variations. The present paper specifically introduces a two-step methodology to select automatically the location of the knots. It proposes to derive an initial model, using nonlinear subspace identification, and incorporating cubic spline basis functions with fixed and equally-spaced abscissas. In a second step, the location of the knots is optimized iteratively by minimizing a least-squares cost function. A single-degree-of-freedom system with a discontinuous stiffness characteristic is considered as a case study.
\end{abstract}

Keywords: nonlinear system identification, frequency-domain subspace algorithm, cubic splines, nonsmooth nonlinearity, automatic splines discretization, constrained nonlinear optimization

\section{Introduction}

The complete process of identifying a nonlinear structural model is often performed using the well-known methodology discussed in [1]. In this methodology, the nonlinear parameter estimation is preceded by a detection and a characterization of the nonlinearity. Such characterization step is generally useful to select an appropriate set of basis functions for the proper estimation, but is not always achievable due to the potential complexity of nonlinear phenomena. In that case, the identification can be carried out in the framework of grey-box modeling where no mathematical representation of the nonlinearity is available, while still assuming a general model structure [2].

In this framework, adopting cubic splines as basis functions proved efficient [3][4]. However, while providing a simple and flexible tool, cubic splines are inherently smooth functions as their first and second derivatives are imposed to be continuous functions. Therefore, the use of cubic splines must be considered with care when considering complex nonlinearities that exhibit discontinuous and nonsmooth behaviors. Such cases can be successfully handled though, if the splines are sufficiently discretized in the regions where abrupt and nonsmooth variations occur [5]. As the knowledge of those regions is rarely available prior to the identification, an automatic procedure is hence needed to find the optimal location of the splines control points, termed knots. The objective of this paper is hence to develop a methodology to address the identification of complex nonlinearities by means of cubic splines whose knot distribution is automatically optimized. The methodology is developed in Section 1 and divided in two steps. First, an initial estimation of the linear and the nonlinear parameters is acquired using nonlinear subspace identification with splines whose knots have fixed and equallyspaced abscissas. This initial estimation is also enhanced by an additional curve fitting operation. Second, that estimation is improved by allowing the knots to move along the $\mathrm{x}$-axis and using an optimization algorithm in order to minimize the model error. Finally, the whole procedure is applied to a system of one degree-of-freedom (DOF) with a piecewise-linear spring, in Section 2, where several cases of knot numbers are considered. 


\section{Identification procedure}

\subsection{Initial state-space model obtained using nonlinear subspace identification and curve fitting}

The first step of the procedure consists in obtaining an initial model that will be later needed for the optimization. This can be performed by means of the Frequency-domain Nonlinear Subspace Identification (FNSI) method [6]. That method enables identifying a state-space model of the system and nonlinear restoring forces defined as the sum of basis functions associated with nonlinear coefficients. In the present case, the basis functions are cubic splines described by the abscissas of their knots. Without any prior knowledge on the nonlinear behavior, the knots are set following an even distribution. The result of the FNSI identification can next be adopted as an initial state for the following optimization process.

However, such initial state often leads to local minima during the optimization, a better state, closer to the optimal solution must hence be provided. Such initial state is obtained by a curve-fitting method where the previously identified nonlinear curve is approximated by a piecewise-linear function. That function possesses a number of linear portions equal to the number of knots minus 1 , and the abscissas of the discontinuities between the linear parts is optimized using a least-square algorithm. Then, the computed abscissas are used as new locations for the splines knots and a new nonlinear curve can be identified. The process can be repeated for a few iterations and the set of abscissas leading to the smallest model error can be selected as an initial state for the full optimization.

\subsection{Final state-space model obtained using nonlinear optimization}

Starting from the initial state defined in the previous section, a nonlinear optimization is carried out on the location of the splines knots using an algorithm of Sequential Quadratic Programming, as it is suited to optimization with inequality constraints [7]. The selected cost function to minimize is the model error, namely the difference between the modeled output, synthesized using the identified system properties, and the measured output.

In order to ensure the correct definition of splines curves, constraints must be imposed on the knots abscissas. Indeed, the knots must be in a crescent order and cannot share the same abscissa. Moreover, the two extreme abscissas are fixed to the minimum and maximum values of the relative displacement while all the others must stay between those two values.

\section{Numerical demonstration on a single-DOF system with a trilinear stiffness}

The described identification procedure is here demonstrated on a single-DOF system whose linear and nonlinear parameters are listed in Table 1. The system possesses a piecewise-linear spring defined by three linear parts having a slope of $k_{n l}$. The transition between those parts occurs at displacements $x_{n l}$ and is regularized using third-order Hermit interpolation polynomials over a range of $\delta * x_{n l}$ to avoid convergence issues during the time integration.

\begin{tabular}{ll|l}
\multicolumn{2}{c|}{ Linear parameters } & Nonlinear parameters \\
\hline$m=2 \mathrm{~kg}$ & $f_{n}=35.59 \mathrm{~Hz}$ & $x_{n l}= \pm 0.01 \mathrm{~m}$ \\
$c=10 \mathrm{Ns} / \mathrm{m}$ & $\varepsilon=1.12 \%$ & $k_{n l}=[50 ; 0 ; 50] \mathrm{kN} / \mathrm{m}$ \\
$k=100 \mathrm{kN} / \mathrm{m}$ & & $\delta=2 \%$
\end{tabular}

Table 1: Linear and nonlinear parameters of the single-DOF system with a trilinear stiffness

A random phase multisine excitation [8] with a flat amplitude spectrum between 5 and $150 \mathrm{~Hz}$ and an RMS value of $100 \mathrm{~N}$ is applied to the system. The time simulation is conducted using a nonlinear Newmark scheme, with a sampling frequency set first to $15000 \mathrm{~Hz}$ for accuracy, and then reduced to $750 \mathrm{~Hz}$ after the integration for practical reasons. 25 periods of 8192 points are considered, including 5 periods to capture transient effects and to remove a posteriori. 


\subsection{Identification using 6-knot splines}

The first considered case tackles the identification with splines possessing 6 knots. Splines with equally-spaced knots provide a nonlinear curve, showed in gray in Figure 1, and an error between the modeled and the measured output of $0.48 \%$. The main discrepancies with the exact curve are found around the discontinuities where more knots are needed, and in the extreme regions where less points are measured, making the identification more arduous.

Using the curve fitting procedure on the gray curve leads to a new set of abscissas. The corresponding identification result is plotted in blue. It can be seen that this curve already presents a knot repartition where the density of knots is higher in the nonsmooth regions. However, even if the repartition is better, the model error is still at $0.22 \%$ as the extreme regions of the curve are poorly estimated.

After an optimization using the curve-fitted result as an initial state, the identified nonlinearity (in red) displays a good estimation in both the nonsmooth and the extreme regions. The knots are seen to be even more concentrated around the discontinuity and the model error drops to $0.018 \%$, which is two orders of magnitude lower than the case of equally-spaced knots.

\section{$2.2 \quad$ Identification using 12-knot splines}

The same identification is carried out while considering splines defined by 12 knots. Estimated nonlinear curves are illustrated in Figure 2 and similar observations to the case of fewer knots can be made. Indeed, it can be seen that the knots location resulting from the optimization is well concentrated in the nonsmooth regions.

Practically, 4 nodes cluster around the discontinuity in the negative displacement and 4 other nodes, very close to each other, around the discontinuity in the positive displacement. Regarding the accuracy of the model, the error goes from $0.14 \%$ for equally-spaced knots to $0.014 \%$ for optimally-located knots, gaining one order of magnitude.

\section{Conclusion}

The goal of this work was to establish a nonlinear identification procedure based on cubic splines with automatic discretization to address the case of complex nonlinear behaviors. This two-step procedure, consisting in a nonlinear subspace identification with a curve-fitting phase followed by an optimization, was demonstrated on a single-DOF system possessing a nonsmooth nonlinearity. The nonlinear stiffness curve was shown to be accurately estimated and the model error significantly decreased after the optimization of the splines knots location.

\section{Acknowledgements}

The authors T. Dossogne and J.P. Noël are Research Fellow (FRIA fellowship) and Postdoctoral Researcher, respectively, of the Fonds de la Recherche Scientifique FNRS which is gratefully acknowledged.

\section{Bibliography}

[1] G. Kerschen, K. Worden, A.F. Vakakis, and J.C. Golinval. Past, present and future of nonlinear system identification in structural dynamics. Mechanical Systems and Signal Processing, 20:505-592, 2006.

[2] J.P. Noël, J. Schoukens, and G. Kerschen. Grey-box nonlinear state-space modelling for mechanical vibrations identification. In Proceedings of the IFAC Symposium on System Identification, Beijing, China, 2015.

[3] J-P. Noël, G. Kerschen, E. Foltête, and S. Cogan. Grey-box identification of a nonlinear solar array structure using cubic splines. International Journal of Non-linear Mechanics, 67:106-119, 2014.

[4] E.J. Dempsey and D.T. Westwick. Identification of Hammerstein models with cubic spline nonlinearities. IEEE Transactions on Biomedical Engineering, 51:237-245, 2004.

[5] J.P. Noël and G. Kerschen. Nonparametric subspace identification of nonlinear structures - application to a spacecraft. In 54 th AIAA/ASME/ASCE/AHS/ASC Structures, Structural Dynamics and Materials Conference, Boston, USA, 2013.

[6] J-P. Noël and G. Kerschen. Frequency-domain subspace identification for nonlinear mechanical systems. Mechanical Systems and Signal Processing, 40:701-717, 2013.

[7] P.T. Boggs and J.W. Tolle. Sequential quadratic programming. Acta Numerica, 4:151, 1995.

[8] R. Pintelon and J. Schoukens. System Identification: A Frequency Domain Approach. IEEE Press, Piscataway, NJ, 2001. 


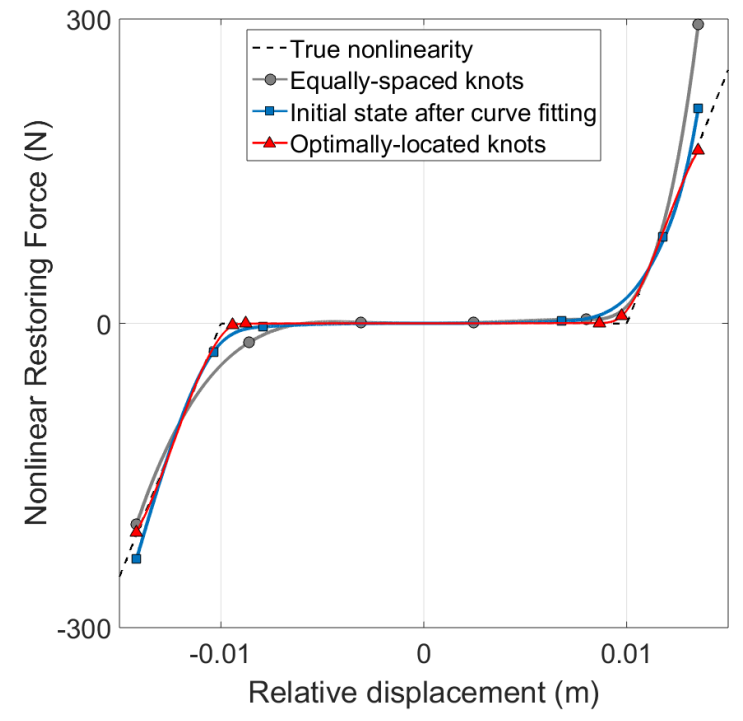

(a)

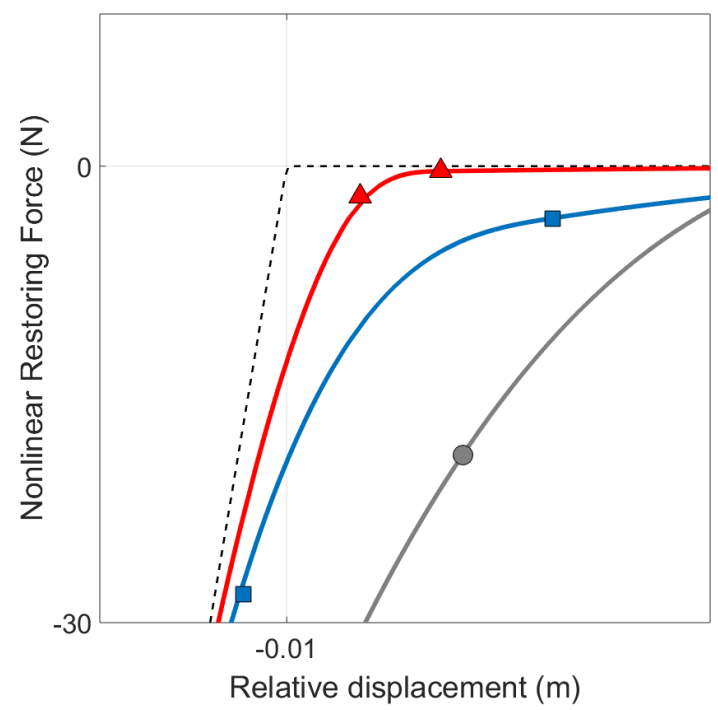

(b)

Figure 1: Exact nonlinear stiffness curve (in dashed line) and FNSI reconstruction using 6-knot cubic splines with: equally-spaced knots (in gray with circles at knots location), knots whose abscissas are found using curve fitting (in blue with squares at knots location) and knots whose abscissas are computed using the complete optimization procedure (in red with triangles at knots location). (a) Entire nonlinear stiffness curve; (b) closeup of the discontinuity in negative displacement

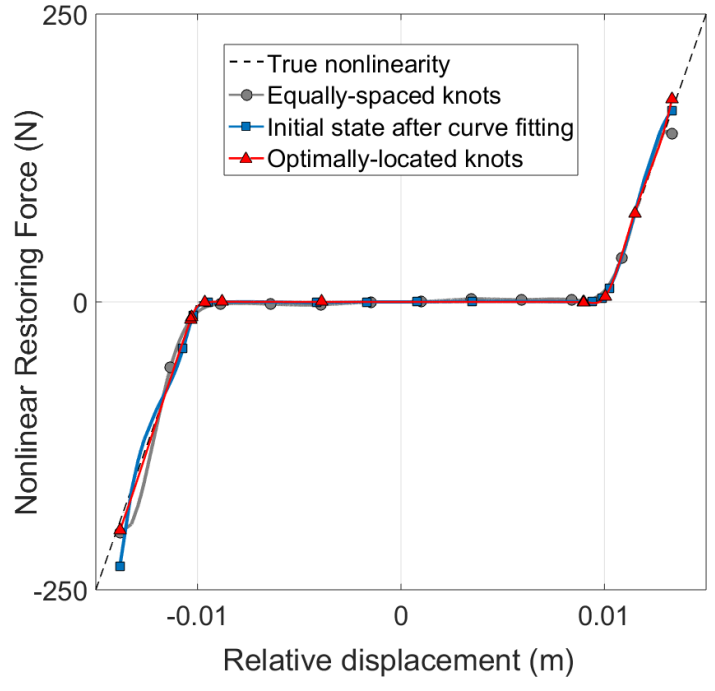

(a)

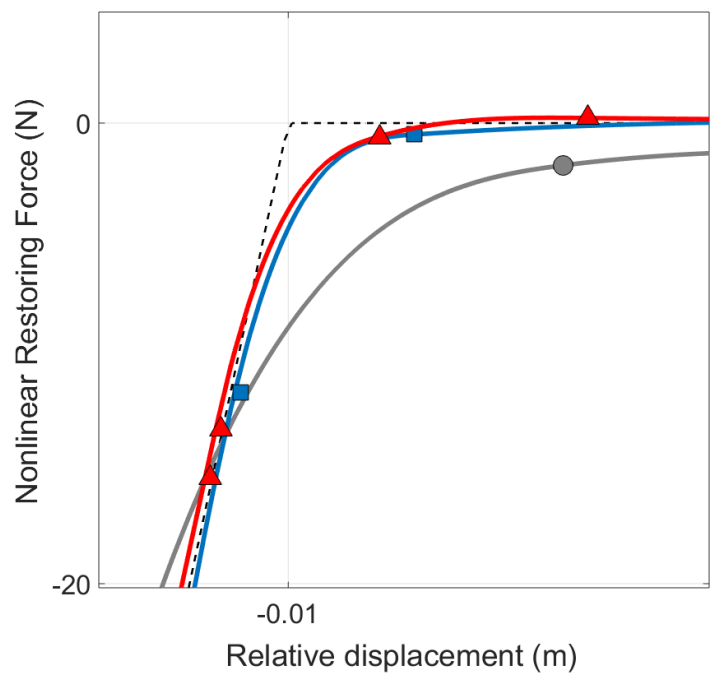

(b)

Figure 2: Exact nonlinear stiffness curve (in dashed line) and FNSI reconstruction using 12-knot cubic splines with: equally-spaced knots (in gray with circles at knots location), knots whose abscissas are found using curve fitting (in blue with squares at knots location) and knots whose abscissas are computed using the complete optimization procedure (in red with triangles at knots location). (a) Entire nonlinear stiffness curve; (b) closeup of the discontinuity in negative displacement 